\title{
Photoimprinted Controllable Fano Resonance in the Terahertz Regime
}

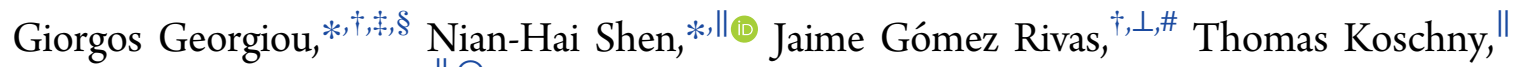
and Costas M. Soukoulis $\|, \bigcirc$

${ }^{\dagger}$ Center for Nanophotonics, AMOLF, Amsterdam, The Netherlands

${ }^{\ddagger}$ CNRS, Inst. NEEL, F-38042 Grenoble, France

${ }^{\S}$ IMEP-LAHC, UMR-CNRS 5130, University of Savoie, 73376 Le Bourget du Lac Cedex, France

"Ames Laboratory and Department of Physics and Astronomy, Iowa State University, Ames, Iowa 50011, United States

${ }^{\perp}$ Dutch Institute for Fundamental Energy Research (DIFFER), Eindhoven, The Netherlands

\#Applied Physics Department, Eindhoven University of Technology, Eindhoven, The Netherlands

OInstitute of Electronic Structure and Laser, FORTH, 71110 Heraklion, Crete Greece

ABSTRACT: The emergence of metamaterials, including the recently proposed metasurfaces, provides unprecedented opportunities in the manipulation as well as the generation of terahertz $(\mathrm{THz})$ waves. Various actively controllable $\mathrm{THz}$ devices such as optical switches and phase modulators have been achieved by incorporating externalstimuli-responsive media (typically semiconductors) in the configurations. Compared to predesigned metallic-resonator-based metamaterials, photoimprinted photonic structures offer us an all-optical route toward reconfigurable functionalities with superior flexibility. Here, we propose to photoimprint some specific patterns on a thin film of semiconductor to excite Fano-like resonances, which result from the coupling between dark and bright elements. Experimental measurements, performed with $\mathrm{THz}$ time-domain spectroscopy, demonstrate counterintuitive tunable deep features in extinction spectra positioned around the expected resonance frequency. Our simulations are in excellent agreement with the experiments, by resembling realistic conditions of low contrast photoimprinted patterns generated with a spatial light modulator and a finite detection time window. This work takes the first step toward realizing switchable Fano resonances via an all-optical approach and, therefore, paves the way to more versatile manipulations of $\mathrm{THz}$ waves.

KEYWORDS: photoimprinting, tunability, Fano resonance, terahertz time-domain spectroscopy

$\mathrm{F}$ or a long time, little attention had been paid to the terahertz $(\mathrm{THz})$ regime, due to the lack of efficient sources and detectors, which leads to the so-called "THz gap". However, the developments of quantum-cascade lasers ${ }^{1}$ and $\mathrm{THz}$ time-domain spectroscopy ${ }^{2}$ have significantly stimulated the interests to this unexplored region of electromagnetic spectrum, which has been found to have various potential applications, such as in security detection, ${ }^{3}$ medical diagnostics, $^{4}$ and communication. ${ }^{5}$ The accomplishment of the promising applications requires substantial development in devices and components for effectively manipulating $\mathrm{THz}$ radiation. Metamaterials, ${ }^{6-9}$ that is, artificially engineered subwavelength composites capable of possessing exotic properties not attainable with natural materials, ${ }^{10-13}$ provide unprecedented possibilities in the generation ${ }^{14}$ and control of $\mathrm{THz}$ waves, ${ }^{15}$ which have led to several devices such as notch filters, ${ }^{16,17}$ absorbers, ${ }^{18}$ and polarizers. ${ }^{19}$ More judiciously, by incorporating materials with properties responsive to external stimuli, for example, semiconductors, as components of metamaterials, we have achieved various dynamically controllable $\mathrm{THz}$ devices, including optical switches, ${ }^{20-22}$ frequency- agile filters, ${ }^{23-25}$ phase modulators, ${ }^{26}$ and plasmonic resonators. $^{27}$ Recently, a more advanced idea of photoimprinted tunable $\mathrm{THz}$ metamaterials has been proposed, ${ }^{28-31}$ in which meta-surfaces are generated via the excitation of carriers by the photo illumination of a semiconductor. These structures can be readily erased via carriers recombination and reconstructed actively, thus, opening new opportunities for a more versatile manipulation of $\mathrm{THz}$ radiation.

Fano resonances, initially described in quantum systems, arise from constructive and destructive interference of a narrow discrete resonance with a broad spectrum or continuum, showing an asymmetric profile of line shape. ${ }^{32}$ In recent years, there have been identified many electromagnetic systems exhibiting a classical analog of Fano resonance, ${ }^{33,34}$ which has attracted much interest due to the enormous applications in energy harvesting, ${ }^{35}$ sensing, ${ }^{36,37}$ lasers, $^{38}$ and nonlinear optics. $^{39}$ In the view of $\mathrm{THz}$ manipulation, tailored Fano

Received: April 12, 2017

Published: June 8, 2017 

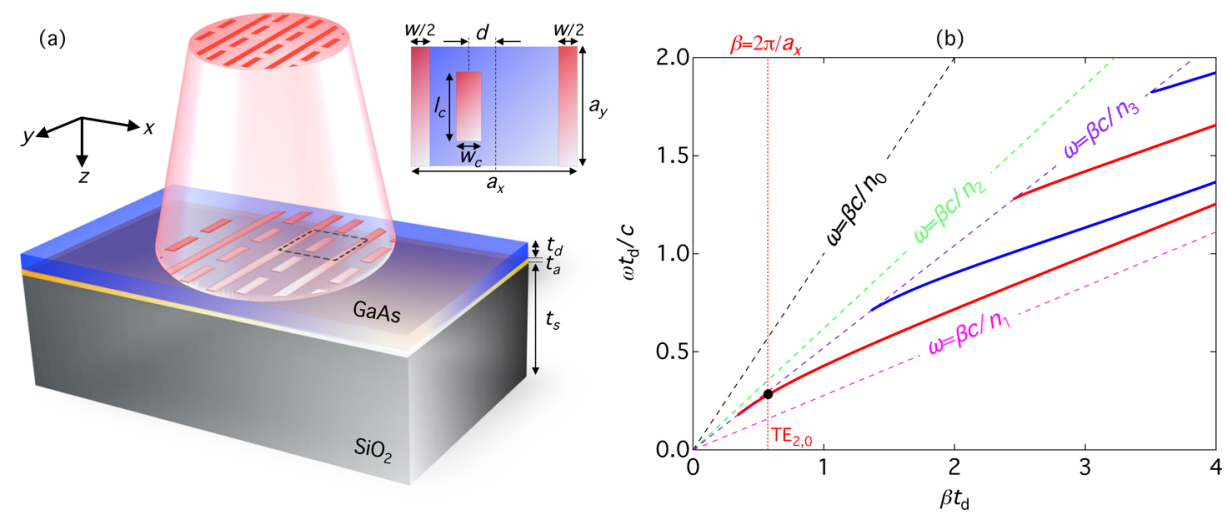

Figure 1. (a) Schematic of the multilayer configuration for actively controlling Fano-like resonances with photoimprinted structures. The inset shows a top view of a photoimprinted unit cell. (b) Dispersion relation of the multilayer system. The vertical dotted line indicates second-order quantized mode along $x$-direction and the solid dot corresponds to $\mathrm{TE}_{2,0}$ eigenstate of GaAs waveguide.

resonant systems may also provide promising opportunities for developing high quality factor devices. There have been reported some related works in this direction, such as actively controllable Fano resonances using metamaterials or metasurfaces made out of predesigned metallic ${ }^{40}$ or graphene ${ }^{41}$ patterns.

In this paper, different from many previously adopted configurations for exciting Fano resonances with prefabricated plasmonic nanostructures ${ }^{42-44}$ or dielectric patterns, ${ }^{45}$ we propose a photoimprinted meta-surface system that exhibits a Fano resonant behavior in the $\mathrm{THz}$ regime. Our experimental demonstration shows a switchable transparency window that is attributed to the excitation of a Fano resonance. By taking into account actual experimental conditions in our simulations, we successfully reproduce the experimental features and reveal two important factors limiting the performance of the proposed photoimprinted tunable device, namely, the imaging contrast ratio and detection length of time window. Our work makes the first attempt in realizing tunable Fano resonance with photoimprinting and opens up opportunities for low-cost and high-efficient control of $\mathrm{THz}$ waves in various ways.

Figure 1a shows the schematic of the proposed multilayer dielectric waveguide configuration under investigation, in which a thin film of GaAs with $t_{\mathrm{d}}=25 \mu \mathrm{m}$ is settled on top of a thick glass substrate $\left(t_{\mathrm{s}} \approx 1 \mathrm{~mm}\right)$. A thin adhesive layer with $t_{\mathrm{a}}=20$ $\mu \mathrm{m}$ glues the GaAs film to the glass substrate. Photo illumination of the sample using light with energy larger than the GaAs band gap energy injects carriers in a specific pattern defined by the illumination (see Figure 1). The unit cell of the design, shown in the inset of Figure $1 \mathrm{a}$, has the period $a_{x}$ and $a_{y}$ along the $x$ - and $y$-direction, respectively, and is composed by side claddings with width $w$ and cut-wire with length $l_{c}$ and width $w_{c}$. The off-center displacement of the cut-wire is denoted by $d$. In this system, the GaAs film is considered as the dark element of the Fano resonator, since the intrinsic waveguide modes are bound states, which do not directly interact with the incoming electromagnetic wave from free space. The conductive cladding regions after photo excitation effectively quantize these waveguide modes, and here, we will utilize the transverse electric (TE) second quantized mode, the so-called $\mathrm{TE}_{2,0}$ mode, which possesses antisymmetric (sineshape within one period) electric field distribution in the $x-y$ plane. The photoimprinted cut wires serve as scatterers interacting with the incident wave and provide a bridge connecting the external wave and the bound state. When the cut wires are displaced away from the center of the unit cell, we should expect some sharp Fano-like resonance features resulting from the interference between the bright and dark modes. In our design, we take the refractive indexes of GaAs, bounding material and substrate to be $n_{1}=3.60, n_{2}=1.61$, and $n_{3}=1.91$, respectively, and the dispersion relations for the two lowest order TE and TM waveguide modes of the GaAs slab in the system are presented in Figure $1 \mathrm{~b}$. By setting the quantization length $a_{x}=275 \mu \mathrm{m}$, the estimated $\mathrm{TE}_{2,0}$ waveguide mode will occur at around $0.54 \mathrm{THz}$. Intuitively, the tunability of the Fano-like feature for the $\mathrm{THz}$ signal can be achieved in the following two ways: one is modulating the optical pump energy to control the conductivity of photoimprinted patterns, and the other is modulating the off-center position of the cut wires.

To experimentally demonstrate the expected tunable Fano resonance via photoimprinting, the desired sample described above is fabricated, with initially a $350 \mu \mathrm{m}$ thick semi-insulating GaAs wafer glued on the quartz substrate, followed by a polishing procedure to achieve the desired $25 \mu \mathrm{m}$ thickness for the GaAs film. It needs to be noted that due to the technical challenges of the polishing process, the resulted sample does not have a homogeneous thickness over the entire two inch diameter. This has been revealed by the measurements using scanning electron microscopy, where a focused ion beam was used to randomly drill holes much smaller than the $\mathrm{THz}$ wavelength on the surface of the sample, showing that the actual $t_{\mathrm{d}}$ varies from $25 \mu \mathrm{m}$ at the outer parts of the wafer to 10 $\mu \mathrm{m}$ near center. However, the focused $\mathrm{THz}$ beam, which is used to probe the optical properties of the sample in the standard $\mathrm{THz}$ time-domain spectroscopy (TDS) setup, has a full-width half-maximum diameter of $2.3 \mathrm{~mm}$, and therefore, we expect that the variation in thickness experienced by the $\mathrm{THz}$ beam, which is settled at the outer region of the sample, will be fairly small, about $0.4 \%$ of the $\mathrm{THz}$ wavelength at $0.5 \mathrm{THz}$.

Our experimental measurements are performed with the optical-pump THz-probe technique, in which, a laser beam delivered by an amplified laser (Coherent Legend Elite HE, 6 $\mathrm{mJ}, 100 \mathrm{fs}$ ) is split into two parts, with a significant fraction $\sim 80 \%$ of the power being used for photo injecting free carriers on the surface of a GaAs film and the rest for the generation and detection of $\mathrm{THz}$ transient waves. The desired photoimprinted patterns are realized with a spatial light modulator (SLM), which is a pixelated liquid crystal device with pixel size as small as $6 \times 6 \mu \mathrm{m}^{2}$. The SLM can change the polarization of 


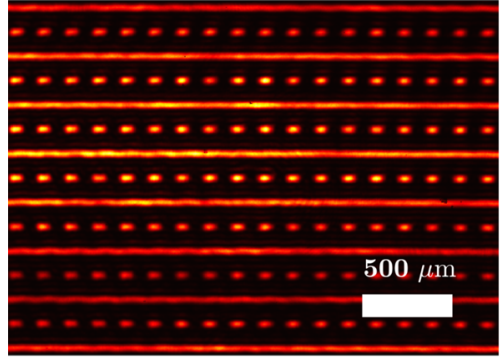

(a)

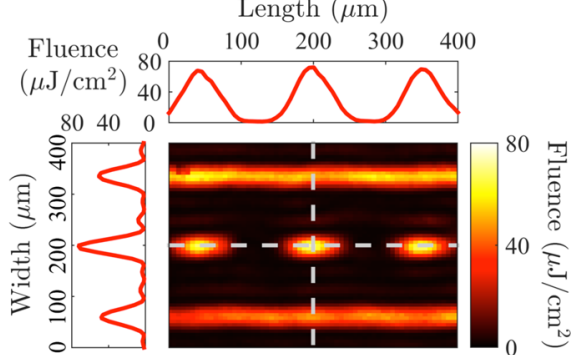

(b)

Figure 2. (a) Photoimprinted image on GaAs film. (b) Zoom-in view of the photoimprinted pattern and the pump fluence along the white dashed cross lines correspondingly.

(a)

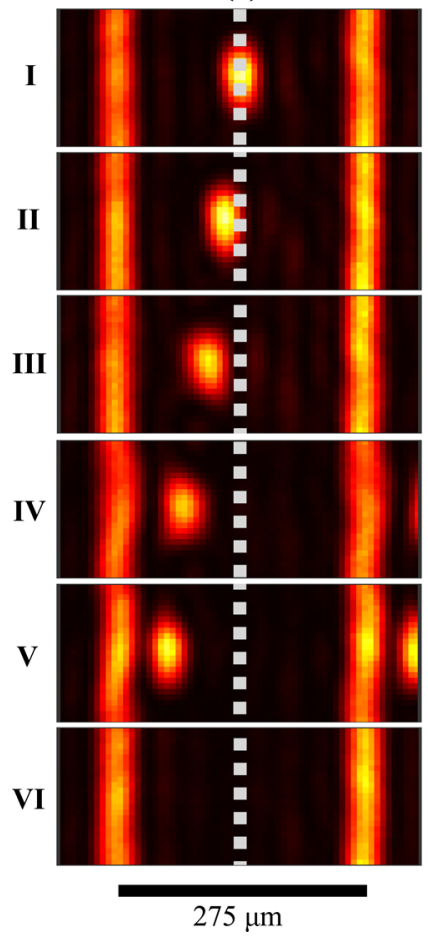

(b)

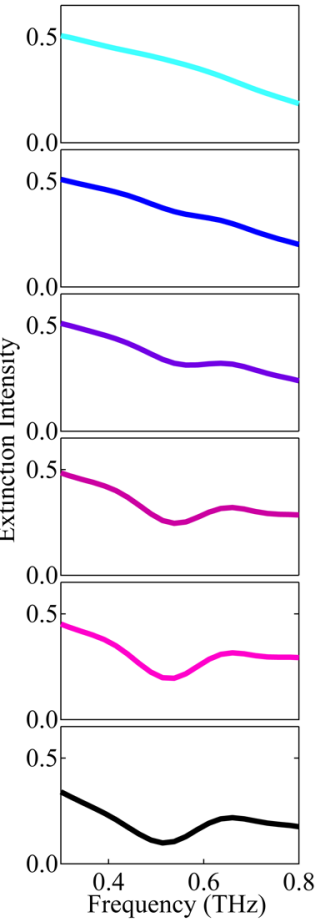

(c)

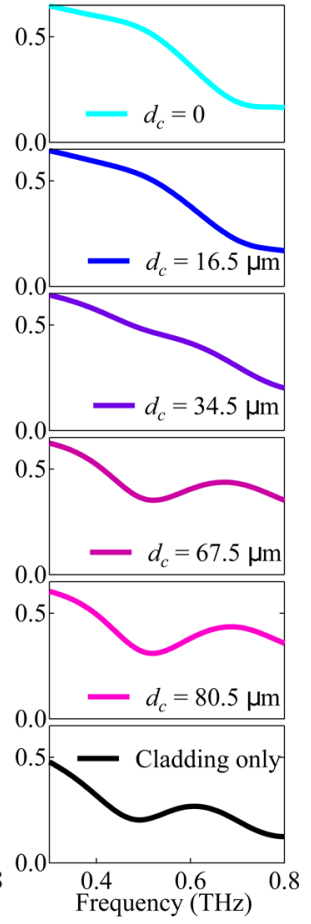

Figure 3. (a) Images of photoimprinted patterns (one unit cell) with different displacements of the cut wires with respect to the cladding lines. (I$\mathrm{V}$ ) and cladding lines only (VI). (b, c) Experimentally measured and postprocessed extinction spectra of the system, respectively, corresponding to (a).

a laser beam, from horizontal to vertical in a continuous way by applying voltage to each individual pixel through the graphics card of a computer. The reflected laser beam goes through a polarizing beam splitter in which white pixels are reflected thus reconstructing the desired image pattern. ${ }^{30}$ Finally, the image is projected on the sample using two plano-convex lenses with focal length $30 \mathrm{~cm}$ each. With respect to the $\mathrm{THz}$ probe beam in our THz TDS system, the involved laser power is then split into two beams, $95 \%$ of the power is used for the generation of $\mathrm{THz}$ pulses in a $0.5 \mathrm{~mm} \mathrm{ZnTe}$ crystal and the remaining $5 \%$ is for the electro-optic detection of $\mathrm{THz}$ pulses in a $1 \mathrm{~mm} \mathrm{ZnTe}$ crystal. The useful bandwidth of the THz spectrum spans from 0.15 to $2.3 \mathrm{THz}$ and the electric field intensity peaks at frequency around $0.5 \mathrm{THz}$ with a signal-to-noise ratio $10^{6}$.

Figure 2a shows an image of the projected pattern, which is captured at the position of the sample by replacing it with a CCD camera. The color scale depicts the level of pump fluence with maximum and minimum values around 80 and $1.5 \mu \mathrm{J} / \mathrm{cm}^{2}$, respectively, as defined by measuring the optical power of the projected beam when the pixels of the SLM are all white or dark correspondingly. The estimated carrier concentration levels are around $4 \times 10^{18}$ and $8 \times 10^{16} \mathrm{~cm}^{-3}$ for white and dark pixel illumination, respectively. Therefore, it should be noted that the carrier concentration with dark pixel illumination is higher than that of the intrinsic GaAs, which is at the level of $10^{15} \mathrm{~cm}^{-3}$ due to the finite intensity rejection of the SLM. As shown in Figure $2 \mathrm{~b}$ for the horizontal and vertical cuts at the top and left side of the projected image, the illuminated units do not have sharp boundaries, as a consequence of the imperfect projection and low numerical aperture of the projection lens. To simplify the analysis of the experimental results, the dimensions of the photoimprinted pattern are estimated based on the optical fluence defining the structures. More specifically, we take effective dimensions by assuming that the boundaries of the structure coincide with the spatial position where the pump intensity is half of its maximum value $\left(I_{0} / 2\right)$. Accordingly, the length and width of cut wires are $l_{\mathrm{c}}=$ $60 \mu \mathrm{m}$ and $w_{\mathrm{c}}=32 \mu \mathrm{m}$, respectively, while for the cladding 
wires we have $a_{y}=155 \mu \mathrm{m}$ and $w=34 \mu \mathrm{m}$. The overall photoimprinted pattern under investigation is positioned in a periodic array with unit cell size $275 \mu \mathrm{m} \times 155 \mu \mathrm{m}$ in the $x-y$ plane.

Upon the generation of the conductive pattern with photo illumination, the probing $\mathrm{THz}$ pulse impinges onto the sample at normal incidence and consequently the zeroth-order transmission signal is collected by an off-axis parabolic mirror, with a collection angle of approximately $8^{\circ}$, and a numerical aperture of 0.14 . The collected transmission signal is detected using the electro-optic sampling method and related to the extinction spectrum by $E=1-T$, where $T$ is the transmission spectrum. The extinction provides useful information about the scattering and absorption cross section of the device. Figure $3 \mathrm{~b}$ shows the measured extinction spectra of the various photoimprinted configurations that correspond to the cut wire being displaced from the center of the unit cell toward the left cladding, as depicted in Figure 3a. When the illuminated cut wires are positioned at the center of the units (see Figure 3a-I), the $\mathrm{TE}_{2,0}$ waveguide mode supported by the GaAs film cannot be excited, and the extinction spectrum appears to be flat without any strong frequency dependence within the band of interest. As the cut wires are gradually shifted off center toward the claddings (from Figure 3a-II to Figure $3 \mathrm{a}-\mathrm{V}$ ), we observe in Figure $3 \mathrm{~b}$ an emerging transparency window around $0.52 \mathrm{THz}$ roughly the Fano resonance position, though the feature is fairly shallow. Upon increasing the off-center displacement of cut wires, the peak transmission eventually reaches the maximum value of approximately $80 \%$.

To understand our experimental results, we have performed numerical simulations using the commercial software CST Microwave Studio. The results, presented in Figure 3c, are in excellent consistency with the experimental results in Figure $3 \mathrm{~b}$. In our simulations, the photo illuminated regions are described with a Drude sheet conductivity model: $\sigma_{\mathrm{s}}=\alpha /(\gamma-i \omega)$, where the collision frequency $\gamma=3.85 \mathrm{THz}$, and the Drude weight $\alpha=$ $9.4 \times 10^{9} \Phi \Omega^{-1} \mathrm{~s}^{-1}$ linearly depending on the pump fluence $\Phi$ in units of $\mu \mathrm{J} / \mathrm{cm}^{2}$. To resemble the actual photoimprinted meta-surface, as shown in Figure 2, we take into account a graded carrier distribution along $x$-direction for the claddings and for the cut wires, we adopt a core-shell pattern, in which the carrier concentration of the core region corresponds to the pump fluence $71 \mu \mathrm{J} / \mathrm{cm}^{2}$ and gradually decreases toward the outer shells. As pointed out above, the finite intensity rejection of the SLM renders the dark image pixels not completely "dark" but with the carrier concentration at the level of $10^{16} \mathrm{~cm}^{-3}$. In our simulations, such a contrast ratio of carrier density between brightest and dark regions is set to be 50, which means the Drude model is applied to dark areas of the photoilluminated pattern with pump fluence approximately $1.4 \mu \mathrm{J} / \mathrm{cm}^{2}$. It has been revealed by our numerical simulations that such a low contrast ratio significantly damps the strength of the expected Fano resonance, leading to the shallow transparency-window features demonstrated in the experiments. An important postprocessing step, namely, signal convolution, has been applied to the transmission spectra by taking into account the limited frequency resolution $(0.125 \mathrm{THz})$ of our $\mathrm{THz}$ setup, as a result of the finite detection time window ( $\sim 8 \mathrm{ps})$. Such a convolution procedure eventually makes the simulations almost perfectly match the experiments. We should also mention that we can rule out the possibility that the measured features result from the dipole resonance of the cut wires by the simulations with different $l_{\mathcal{O}}$ in which no shift of the transparency window is observed.

Finally, we would like to mention that we are also able to achieve a switchable Fano resonance by modulating the pump power in the configuration. For a fixed displacement of cut wires, for example $d=67.5 \mu \mathrm{m}$, at fairly low pump levels, the transparency window corresponding to the Fano resonance gradually builds up upon the increase of pump power. However, at very high pump levels, the carrier densities in the dark regions will increase dramatically due to the limited contrast ratio of the setup, and it leads to the decrease of the overall transmission for the configuration.

In this paper, we experimentally demonstrate all-optically controlled Fano resonances in the $\mathrm{THz}$ regime, showing a switchable transparency window, which has been successfully reproduced in simulations. It is revealed that the shallow features in our experiments are due to the low contrast ratio of the illuminated pattern by a spatial light modulator and the short time window of the $\mathrm{THz}$ time-domain spectroscopy system. By taking into consideration these two aspects, the performance of such photoimprinted tunable devices can be improved accordingly. Our work makes an initial effort to take advantage of Fano resonances for all-optical high speed tunability, and broadens the view of $\mathrm{THz}$ control in more versatile ways.

\section{AUTHOR INFORMATION}

\section{Corresponding Authors}

*E-mail: giorgos.georgiou@neel.cnrs.fr.

*E-mail: nhshen@ameslab.gov.

\section{ORCID}

Nian-Hai Shen: 0000-0002-9715-0130

\section{Notes}

The authors declare no competing financial interest.

\section{ACKNOWLEDGMENTS}

This work was supported by the U.S. Department of Energy (DOE), Office of Science, Basic Energy Science, Materials Sciences and Engineering Division. The research was performed at Ames Laboratory, which is operated for the U.S. DOE by Iowa State University under Contract No. DEAC02-07CH11358. This work was also supported by the U.S. Office of Naval Research Award No. N00014-14-1-0474 (simulations) and by ERC Grant No. 320081 (PHOTOMETA; theory). This work has been supported by the ERC through Grant No. 259727 THZ-PLASMON. This work is part of the research programme of The Netherlands Organisation for Scientific Research (NWO).

\section{REFERENCES}

(1) Grischkowsky, D.; Keiding, S.; van Exter, M.; Fattinger, C. Farinfrared time-domain spectroscopy with terahertz beams of dielectrics and semiconductors. J. Opt. Soc. Am. B 1990, 7, 2006-2015.

(2) Köhler, R.; Tredicucci, A.; Beltram, F.; Beere, H. E.; Linfield, E. H.; Davies, A. G.; Ritchie, D. A.; Iotti, R. C.; Rossi, F. Terahertz semiconductor-heterostructure laser. Nature 2002, 417, 156-159.

(3) Federici, J. F.; Schulkin, B.; Huang, F.; Gary, D.; Barat, R.; Oliveira, F.; Zimdars, D. THz imaging and sensing for security applications-explosives, weapons and drugs. Semicond. Sci. Technol. 2005, 20, S266-S280.

(4) Siegel, P. Terahertz technology in biology and medicine. IEEE Trans. Microwave Theory Tech. 2004, 52, 2438-2447. 
(5) Fitch, M. J.; Osiander, R. Terahertz waves for communications and sensing. Johns Hopkins APL Technol. Dig. 2004, 25, 348-355.

(6) Shelby, R. A.; Smith, D. R.; Schultz, S. Experimental Verification of a Negative Index of Refraction. Science 2001, 292, 77-79.

(7) Smith, D. R.; Pendry, J. B.; Wiltshire, M. C. Metamaterials and negative refractive index. Science 2004, 305, 788-792.

(8) Soukoulis, C. M.; Wegener, M. Past achievements and future challenges in the development of three-dimensional photonic metamaterials. Nat. Photonics 2011, 52, 523-530.

(9) Zheludev, N. I.; Kivshar, Y. S. From metamaterials to metadevices. Nat. Mater. 2012, 11, 917-924.

(10) Pendry, J. B. Negative refraction makes a perfect lens. Phys. Rev. Lett. 2000, 85, 3966-3969.

(11) Fang, N. Sub-Diffraction-Limited Optical Imaging with a Silver Superlens. Science 2005, 308, 534-537.

(12) Schurig, D.; Mock, J. J.; Justice, B. J.; Cummer, S. A.; Pendry, J. B.; Starr, A. F.; Smith, D. R. Metamaterial electromagnetic cloak at microwave frequencies. Science 2006, 314, 977-980.

(13) Fang, A.; Koschny, T.; Soukoulis, C. M. Optical anisotropic metamaterials: Negative refraction and focusing. Phys. Rev. B: Condens. Matter Mater. Phys. 2009, 79, 245127.

(14) Luo, L.; Chatzakis, I.; Wang, J.; Niesler, F. B. P.; Wegener, M.; Koschny, T.; Soukoulis, C. M. Broadband terahertz generation from metamaterials. Nat. Commun. 2014, 5, 3055.

(15) Yen, T. J. Terahertz Magnetic Response from Artificial Materials. Science 2004, 303, 1494-1496.

(16) Chen, H.-T.; O’Hara, J. F.; Taylor, A. J.; Averitt, R. D.; Highstrete, C.; Lee, M.; Padilla, W. J. Complementary planar terahertz metamaterials. Opt. Express 2007, 15, 1084-1095.

(17) Tao, H.; Strikwerda, A. C.; Fan, K.; Padilla, W. J.; Zhang, X.; Averitt, R. D. Reconfigurable Terahertz Metamaterials. Phys. Rev. Lett. 2009, 103, 147401.

(18) Landy, N. I.; Sajuyigbe, S.; Mock, J. J.; Smith, D. R.; Padilla, W. J. Perfect metamaterial absorber. Phys. Rev. Lett. 2008, 100, 207402.

(19) Ren, L.; Pint, C. L.; Arikawa, T.; Takeya, K.; Kawayama, I.; Tonouchi, M.; Hauge, R. H.; Kono, J. Broadband terahertz polarizers with ideal performance based on aligned carbon nanotube stacks. Nano Lett. 2012, 12, 787-790.

(20) Janke, C.; Rivas, J. G.; Bolivar, P. H.; Kurz, H. All-optical switching of the transmission of electromagnetic radiation through subwavelength apertures. Opt. Lett. 2005, 30, 2357-2359.

(21) Chen, H.-T.; Padilla, W. J.; Zide, J. M. O.; Bank, S. R.; Gossard, A. C.; Taylor, A. J.; Averitt, R. D. Ultrafast optical switching of terahertz metamaterials fabricated on ErAs/GaAs nanoisland superlattices. Opt. Lett. 2007, 32, 1620-1622.

(22) Padilla, W. J.; Taylor, A. J.; Highstrete, C.; Lee, M.; Averitt, R. D. Dynamical Electric and Magnetic Metamaterial Response at Terahertz Frequencies. Phys. Rev. Lett. 2006, 96, 107401.

(23) Chen, H.-T.; O’Hara, J. F.; Azad, A. K.; Taylor, A. J.; Averitt, R. D.; Shrekenhamer, D. B.; Padilla, W. J. Experimental demonstration of frequency-agile terahertz metamaterials. Nat. Photonics 2008, 2, 295298.

(24) Shen, N.-H.; Massaouti, M.; Gokkavas, M.; Manceau, J.-M.; Ozbay, E.; Kafesaki, M.; Koschny, T.; Tzortzakis, S.; Soukoulis, C. M. Optically Implemented Broadband Blueshift Switch in the Terahertz Regime. Phys. Rev. Lett. 2011, 106, 037403.

(25) Fan, K.; Strikwerda, A. C.; Zhang, X.; Averitt, R. D. Threedimensional broadband tunable terahertz metamaterials. Phys. Rev. B: Condens. Matter Mater. Phys. 2013, 87, 161104.

(26) Chen, H.-T.; Padilla, W. J.; Cich, M. J.; Azad, A. K.; Averitt, R. D.; Taylor, A. J. A metamaterial solid-state terahertz phase modulator. Nat. Photonics 2009, 3, 148-151.

(27) Berrier, A.; Ulbricht, R.; Bonn, M.; Gómez Rivas, J.; Chen, H.-t.; Azad, A. K.; Taylor, A. J.; Averitt, R. D.; Shrekenhamer, D. B.; Padilla, W. J.; Cich, M. J. Ultrafast active control of localized surface plasmon resonances in silicon bowtie antennas. Opt. Express 2010, 18, 2322623235.

(28) Okada, T.; Tanaka, K. Photo-designed terahertz devices. Sci. Rep. 2011, 1, 121.
(29) Chatzakis, I.; Tassin, P.; Luo, L.; Shen, N.-H.; Zhang, L.; Wang, J.; Koschny, T.; Soukoulis, C. M. One- and two-dimensional photoimprinted diffraction gratings for manipulating terahertz waves. Appl. Phys. Lett. 2013, 103, 043101.

(30) Georgiou, G.; Tyagi, H. K.; Mulder, P.; Bauhuis, G. J.; Schermer, J. J.; Rivas, J. G. Photo-generated $\mathrm{THz}$ antennas. Sci. Rep. 2015, 4, 3584.

(31) Steinbusch, T. P.; Tyagi, H. K.; Schaafsma, M.; Georgiou, G.; Gómez Rivas, J. Active terahertz beam steering by photo-generated graded index gratings in thin semiconductor films. Opt. Express 2014, 22, 26559.

(32) Fano, U. Effects of configuration interaction on intensities and phase shifts. Phys. Rev. 1961, 124, 1866-1878.

(33) Luk'yanchuk, B.; Zheludev, N. I.; Maier, S. A.; Halas, N. J.; Nordlander, P.; Giessen, H.; Chong, C. T. The Fano resonance in plasmonic nanostructures and metamaterials. Nat. Mater. 2010, 9, 707-715.

(34) Miroshnichenko, A. E.; Flach, S.; Kivshar, Y. S. Fano resonances in nanoscale structures. Rev. Mod. Phys. 2010, 82, 2257-2298.

(35) Wu, C.; Khanikaev, A. B.; Adato, R.; Arju, N.; Yanik, A. A.; Altug, H.; Shvets, G. Fano-resonant asymmetric metamaterials for ultrasensitive spectroscopy and identification of molecular monolayers. Nat. Mater. 2011, 11, 69-75.

(36) Adato, R.; Yanik, A. A.; Amsden, J. J.; Kaplan, D. L.; Omenetto, F. G.; Hong, M. K.; Erramilli, S.; Altug, H. Ultra-sensitive vibrational spectroscopy of protein monolayers with plasmonic nanoantenna arrays. Proc. Natl. Acad. Sci. U. S. A. 2009, 106, 19227-19232.

(37) Kabashin, A. V.; Evans, P.; Pastkovsky, S.; Hendren, W.; Wurtz, G. A.; Atkinson, R.; Pollard, R.; Podolskiy, V. A.; Zayats, A. V. Plasmonic nanorod metamaterials for biosensing. Nat. Mater. 2009, 8, $867-71$.

(38) Zheludev, N. I.; Prosvirnin, S. L.; Papasimakis, N.; Fedotov, V. A. Lasing spaser. Nat. Photonics 2008, 2, 351-354.

(39) Fan, P.; Yu, Z.; Fan, S.; Brongersma, M. L. Optical Fano resonance of an individual semiconductor nanostructure. Nat. Mater. 2014, 13, 471-475.

(40) Gu, J.; Singh, R.; Liu, X.; Zhang, X.; Ma, Y.; Zhang, S.; Maier, S. A.; Tian, Z.; Azad, A. K.; Chen, H.-T.; Taylor, A. J.; Han, J.; Zhang, W. Active control of electromagnetically induced transparency analogue in terahertz metamaterials. Nat. Commun. 2012, 3, 1151.

(41) Zhang, Y.; et al. A graphene based tunable terahertz sensor with double Fano resonances. Nanoscale 2015, 7, 12682-12688.

(42) Hao, F.; Sonnefraud, Y.; Dorpe, P. V.; Maier, S. A.; Halas, N. J.; Nordlander, P. Symmetry Breaking in Plasmonic Nanocavities: Subradiant LSPR Sensing and a Tunable Fano Resonance. Nano Lett. 2008, 8, 3983-3988.

(43) Verellen, N.; Sonnefraud, Y.; Sobhani, H.; Hao, F.; Moshchalkov, V. V.; Dorpe, P. V.; Nordlander, P.; Maier, S. A. Fano Resonances in Individual Coherent Plasmonic Nanocavities. Nano Lett. 2009, 9, 1663-1667.

(44) Fan, J. A.; Wu, C.; Bao, K.; Bao, J.; Bardhan, R.; Halas, N. J.; Manoharan, V. N.; Nordlander, P.; Shvets, G.; Capasso, F. SelfAssembled Plasmonic Nanoparticle Clusters. Science 2010, 328, 11351138.

(45) Yang, Y.; Kravchenko, I. I.; Briggs, D. P.; Valentine, J. Alldielectric metasurface analogue of electromagnetically induced transparency. Nat. Commun. 2014, 5, 5753. 